\title{
Hadronic modes and quark properties in the quark-gluon plasma
}

\author{
M. Mannarelli* and R. Rapp \\ Cyclotron Institute and Physics Department, Texas A\&M University, College Station, Texas 77843-3366, USA
}

(Received 16 May 2005; published 15 December 2005)

\begin{abstract}
Based on interaction potentials between a heavy quark and antiquark as extracted from recent QCD lattice calculations, we set up a Brueckner-type many-body scheme to study the properties of light (anti-) quarks in a quark-gluon plasma at moderate temperatures, $T \simeq 1-2 T_{c}$. The quark-antiquark $T$ matrix, including both color-singlet and color-octet channels, and corresponding quark self-energies and spectral functions are calculated self-consistently. The repulsive octet potential induces quasiparticle masses of up to $150 \mathrm{MeV}$, whereas the attractive color-singlet part generates resonance structures in the $q-\bar{q} T$ matrix, which in turn lead to quasiparticle widths of $\sim 200 \mathrm{MeV}$. This corresponds to scattering rates of $\sim 1 \mathrm{fm}^{-1}$ and may reflect liquid-like properties of the system.
\end{abstract}

DOI: 10.1103/PhysRevC.72.064905

PACS number(s): 25.75.Dw, 12.38.Gc, 24.85.+p, 25.75.Nq

\section{INTRODUCTION}

A central goal of the relativistic heavy-ion collision program is the creation and identification of new forms of highly excited nuclear matter, in particular a deconfined and chirally symmetric quark-gluon plasma (QGP). At sufficiently high temperature $T$, owing to asymptotic freedom of quantum chromodynamics (QCD), the QGP is expected to be a weakly interacting gas of quark and gluon quasiparticles with comparatively small thermal masses, $m_{q, g} \sim g T$. Recent data from the Relativistic Heavy-Ion Collider (RHIC) indicate, however, that the produced matter exhibits strong collective behavior that is incompatible with a weakly interacting QGP: Standard $(2 \leftrightarrow 2)$ perturbative QCD (pQCD) cross sections for quarks and gluons do not allow for rapid thermalization [1] as required in hydrodynamic models to reproduce the observed magnitude of the elliptic flow [2-4]. The estimated initial energy densities well in excess of the critical one predicted by lattice $\mathrm{QCD}(\mathrm{lQCD}), \epsilon_{c} \simeq 1 \mathrm{GeV} / \mathrm{fm}^{3}$, raises questions about the nature of the produced medium at temperatures $T \simeq 1-2 T_{c}$ (where $T_{c} \simeq 170 \mathrm{MeV}$ is the critical temperature). Of particular importance is the identification of the relevant interactions that can lead to sufficiently large scattering rates while maintaining consistency with the QGP equation of state (EoS), as determined in lQCD.

Recent (quenched) 1QCD calculations found intriguing evidence that mesonic correlation functions, after transformation into Minkowski space, exhibit resonance-like (or bound-state-like) structures for temperatures up to $\sim 2 T_{c}$. This was first observed for low-lying charmonia $\left(\eta_{c}, J / \psi\right)$ [5-7], but subsequently also for mesonic systems with lighter quarks [8,9]. As is well known, resonance scattering is typically characterized by isotropic angular distributions and thus is more efficient in randomizing momentum distributions than forward-dominated pQCD cross sections. Indeed, a recent calculation [10] based on the assumption of resonant

\footnotetext{
*Present address: Center for Theoretical Physics, Laboratory for Nuclear Science and Department of Physics, Massachusetts Institute of Technology, Cambridge, MA 02139.
}

" $D$ "-meson states in the QGP has shown that thermal relaxation times for charm quarks are reduced by a factor of $\sim 3$ as compared to using perturbative rescattering cross sections. The possibility of light hadronic states (especially for the pion and its chiral partner $\sigma$ ) surviving above the phase transition has been suggested some time ago using effective quark interactions, for example, within the Nambu-Jona-Lasinio model [11,12], within the instanton-liquid model based on Euclidean correlators [13], or more recently in Refs. [14,15].

To make closer contact to $1 Q C D$, some recent works have extracted a (color-singlet) heavy-quark $(Q-\bar{Q})$ potential $V_{1}$, from the corresponding lQCD free energy $F_{1}$, at finite $T$, and injected it into a Schrödinger equation to infer quarkonium properties [16-18]. Reasonable consistency was found in that the heavy-quark bound states dissolve at roughly the same temperatures at which the peaks in the lQCD spectral functions disappear $\left(\sim 2 T_{c}\right.$ for $J / \psi$ and $\left.\eta_{c}\right)$, provided the free energy was converted into a potential by subtracting an entropy term according to $V_{1}=F_{1}-T d F_{1} / d T$. A similar approach has also been applied to the light-quark sector in Refs. [19-21], where the $q-\bar{q}$ potentials from unquenched 1QCD (including colored channels) have been supplemented by relativistic (and instanton-induced) interaction corrections. By assuming rather large quark- and gluon-quasiparticle masses, $m_{q, g} \simeq(3-4) T$ (motivated by 1QCD calculations of temporal masses [22]), light mesonic, as well as a large number of colored diquark, quark-gluon, and gluon-gluon, bound states have been found. Both quark and gluon quasiparticles and binary bound states together were shown to approximately reproduce the EoS from IQCD. However, the effects of finite widths for both quarks, antiquarks and bound states, which are essential to address scattering problems, were not included.

In the present article we employ quark-antiquark potentials extracted from 1QCD (including relativistic corrections as in Refs. [19-21]) within a three-dimensionally reduced BetheSalpeter equation to evaluate quark and antiquark interactions in the QGP. We compute the pertinent scattering $(T)$ matrices in both color-singlet and color-octet channels and calculate the quark self-energies including both real and imaginary parts (corresponding to quasiparticle masses and widths). The self-energies, in turn, are reinserted into the $q-\bar{q}$ propagator of 

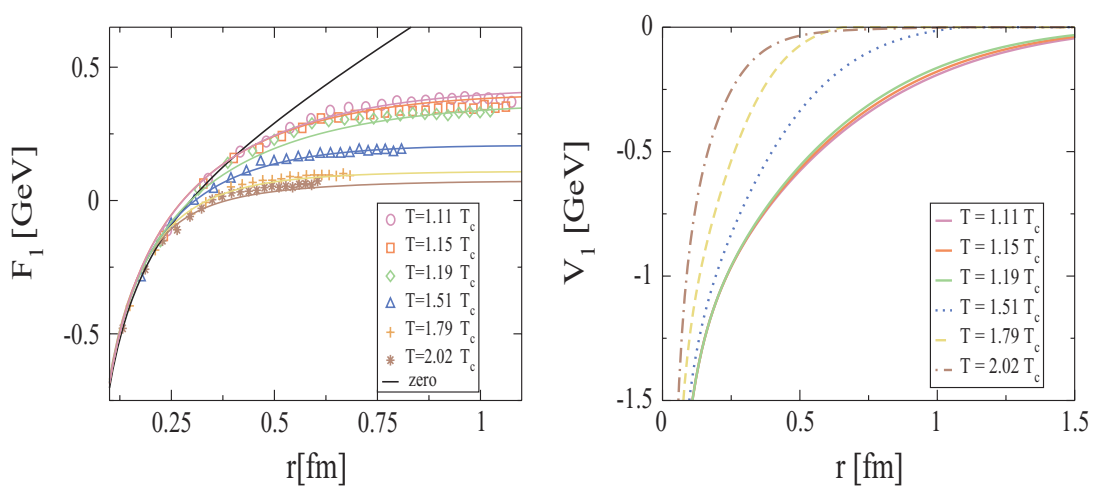

FIG. 1. (Color online) Left panel: Lattice QCD results for the color-singlet free energy from unquenched simulations [27] for six different values of the temperature (symbols) compared to our fit function, Eq. (1), represented by the various curves. Right panel: Corresponding potential in the color-singlet channel obtained with Eq. (5) for the six different values of the temperature. the $T$-matrix equation, constituting a self-consistency problem, which we solve by numerical iteration. We comment on possible consequences of our results for quasiparticle masses and widths with respect to the QGP EoS and (anti-) quark rescattering time scales, respectively.

Our article is organized as follows. In Sec. II we present our parametrization of 1QCD data for the singlet free energy and extract a pertinent quark-antiquark potential including both color-singlet and color-octet contributions. In Sec. III we set up our self-consistency problem comprising the $q-\bar{q}$ scattering equation and in-medium single-particle self-energies and propagators and discuss the underlying assumptions and approximations. The numerical results with accompanying discussion for the $T$ matrix and self-energy in a nonperturbative QGP are contained in Sec. IV. In Sec. V we conclude and give an outlook.

\section{QUARK-ANTIQUARK POTENTIAL FROM LATTICE QCD}

To obtain a driving term (potential) for a $q-\bar{q}$ scattering equation we take recourse to $1 \mathrm{QCD}$ calculations of the static free energy for a $Q-\bar{Q}$ pair. The Bielefeld group has performed extensive studies of this quantity based on Polyakov loop correlators [23] for both the pure-glue SU(3) [24,25] and $N_{f}=2$ QCD [26,27]. Various parametrizations thereof have been given in the literature (e.g., Refs. [17,28-30]). For the temperature range $T=(1.1-2) T_{c}$, it turns out that unquenched singlet free energy [26,27] can be reasonably well reproduced by the the following form reminiscent of a screened Cornell potential (as suggested, e.g., in Ref. [28]):

$$
F_{1}(r, T)=-\frac{\alpha}{r} e^{-a \mu(r, T) r}+\frac{\sigma}{\mu(r, T)}\left(1-e^{-\mu(r, T) r}\right),
$$

with a "screening mass"

$$
\mu(r, T)=\frac{\sigma}{b} e^{-0.3 / r}
$$

and two fitting functions given by

$$
\begin{aligned}
& a \equiv a(r, T)=\frac{1}{2 \sqrt{\mu(r, T)}}, \\
& b \equiv b(t)=1.1-3.6 T-4.3 T^{2}+17.5 T^{3},
\end{aligned}
$$

where $\alpha=0.4$, and $\sigma=1.2 \mathrm{GeV}^{2}$. The left panel of Fig. 1 summarizes our fit to the lattice "data." Also shown is the unquenched zero-temperature potential as obtained in Ref. [29] (recall that for $T=0, E_{1}=F_{1}$, see also the following), which is used to normalize the finite- $T$ results at short distances, $r<0.2 \mathrm{fm}$, where the free energy is no longer expected to depend on temperature. Our parametrization, Eq. (1), accommodates this $T=0$ constraint.

As mentioned in Sec. I, the appropriate quantity in relation to the free energy that can serve as an effective potential appears to be the (color-singlet) internal energy $E_{1}$. Following Kaczmarek et al. [25], we subtract the entropy contribution to the free energy according to

$$
E_{1}=F_{1}-T \frac{d F_{1}}{d T} .
$$

The nonzero asymptotic value of the internal energy can now be interpreted as an in-medium quark mass that should not be included in the interaction part of the potential. One therefore assumes that the potential in the color-singlet channel can be extracted via

$$
V_{1}(r, T)=E_{1}(r, T)-E_{1}(\infty, T) .
$$

The singlet potential is shown in the right panel of Fig. 1 for the same values of temperature as the singlet free-energy (left panel). The potentials are appreciably larger in magnitude than the corresponding free energies and decrease with increasing temperature.

To illustrate uncertainties in the determination of the potentials we compare in Fig. 2 our results with the ones obtained by other groups for temperatures of $1.5 T_{c}$ (left panel) and $2 T_{c}$ (right panel). Although the potentials of Refs. [17] (Wo) and [18] (MP) are extracted from quenched 1QCD, the one of Ref. [21] (SZ) and ours (MR) result from unquenched simulations. At $1.5 T_{c}$ our potential is about $30-40 \%$ more attractive than that of SZ at distances between 0.1 and $0.8 \mathrm{fm}$, whereas similar deviations also occur between the quenched-based results; however, at $2 T_{c}$ our potential is less attractive than both the quenched (Wo) and unquenched (SZ) results. We therefore conclude that the current uncertainty in the extraction of the potentials amounts to about $50 \%$ and that it does not yet allow for a systematic discrimination between quenched and unquenched results [provided the temperature dependence is normalized to $T_{c}$, which is, of course, quite different in quenched $(\sim 260 \mathrm{MeV})$ and unquenched 

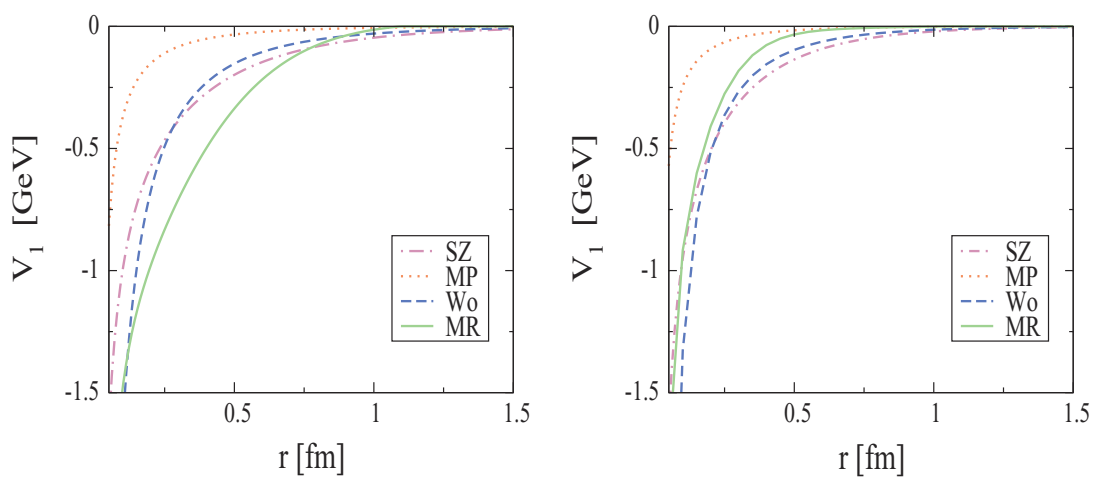

FIG. 2. (Color online) Comparison of $Q-\bar{Q}$ color-singlet potentials as determined from various lattice data in our (MR) and other works (SZ [21], MP [18], and Wo [17]); the left (right) panel is for $T=1.5 T_{c}\left(2 T_{c}\right)$.

( $\sim 170 \mathrm{MeV})$ simulations]. The pertinent uncertainties will be assessed in the following by performing $T$-matrix calculations for charmonium ( $c-\bar{c}$ systems) with both our potential and the quenched Wo potential (cf. Sec. IV A) and comparing them to spectral functions from IQCD [6] obtained with different methods; reasonable agreement will be found.

Toward a more complete description of the $q-\bar{q}$ interactions in the QGP we will in this work also consider the (repulsive) contributions from the color-octet channel. However, as pointed out in Ref. [31], the octet potential cannot be straightforwardly inferred from the Polyakov loop correlators. Because of a lack of better knowledge of the octet free energies, we here assume that the octet potential follows the leading order result of perturbation theory,

$$
F_{8}=-\frac{1}{8} F_{1} \text {. }
$$

Again, we will check the sensitivity of our calculations to this approximation, by varying the coefficient in Eq. (6) by a factor of $0.5-2$.

For nonstatic quarks it is also important to include relativistic corrections [20,32]. Following Ref. [20] we implement a velocity-velocity interaction term by the replacement $V(r) \rightarrow$ $V(r)\left(1-\hat{\alpha}_{1} \cdot \hat{\alpha}_{2}\right)$, where $\hat{\alpha}_{1}$ and $\hat{\alpha}_{2}$ are quasiparticle velocity operators. As pointed out in Ref. [21], this procedure is strictly speaking, correct only for a Coulomb-type potential.

\section{REDUCED BETHE-SALPETER EQUATION, QUARK SELF-ENERGY, AND SELF-CONSISTENCY}

To evaluate quark-antiquark interactions in the QGP we employ the $T$-matrix approach, as is well known from the nuclear many-body problem. In relativistic field theory, the starting point is a four-dimensional Bethe-Salpeter (BS) equation,

$$
T=K+\int K S S T,
$$

where $K$ denotes the interaction kernel and $S$ is the singleparticle propagator. Both quantities carry, in principle, dependencies on temperature and (baryon) density of the surrounding medium. Since the effective $q-\bar{q}$ potential constructed in the previous section is essentially nonrelativistic in nature, it is appropriate to employ the ladder approximation to Eq. (7) in connection with neglecting virtual particle-antiparticle loops. Thus, we will identify the kernel $K$ with the potential $V$ with appropriate approximations in the propagator and scattering equation to be discussed in the following.

The medium effects in the quark propagator $S$ are encoded in a self-energy, which we decompose according to

$$
\Sigma=\tilde{\Sigma}+\int T S
$$

The first term, $\tilde{\Sigma}$, represents a "gluon-induced" contribution from interactions of quarks and antiquarks with surrounding thermal gluons. In this work we do not calculate this term explicitly, but we will study how different (purely real) values affect our results. We note that a perturbative (hard-thermalloop) form of this (mass) term is widely used as a parameter in quasiparticle descriptions of the QGP EoS [33-36]. The second term on the right side of Eq. (8) is the contribution to the self-energy induced by interactions with antiquarks of the heat bath, which we compute at the same level of approximation as the $T$ matrix. In principle, the quark self-energy also receives contributions from interactions with thermal quarks (which could be significant, especially in the scalar diquark channel), but we neglect them in this work. We also constrain ourselves to the case of vanishing quark chemical potential, $\mu_{q}=0$, which implies equal self-energies for quarks and antiquarks. The two equations (7) and (8) constitute a self-consistency problem that is diagrammatically illustrated in Fig. 3.

Let us discuss the single-particle quantities in more detail. The full quark propagator obeys a Schwinger-Dyson equation,

$$
S=S_{0}+S_{0} \Sigma S,
$$

where $S_{0}(k)=\left[\not k-m_{0}\right]^{-1}$. In the following, we set the current quark mass $m_{0}$ to zero and assume the self-energy to take the

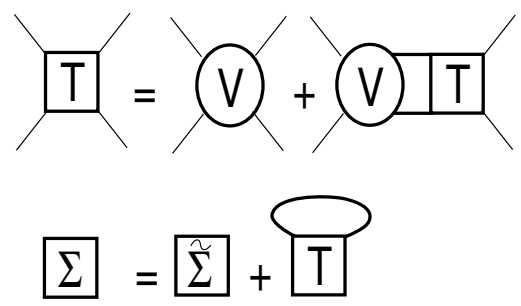

FIG. 3. Schematic representation of the self-consistency problem composed of the Bethe-Salpeter equation (7) in ladder approximation and the quark self-energy, Eq. (8). Thick lines represent full fermionic propagators. The four different blocks correspond to the $T$ matrix $(T)$, potential $(V)$, self-energy $(\Sigma)$ and, "gluon-induced" self-energy $(\widetilde{\Sigma})$. 
(chirally invariant) form

$$
\Sigma(\omega, \mathbf{k})=a(\omega, k) \gamma_{0}+b(\omega, k) \hat{\mathbf{k}} \cdot \gamma
$$

since scalar and tensor contributions are suppressed owing to chiral symmetry restoration (above $T_{c}$ ), whereas pseudoscalar and axial-vector terms are absent owing to parity invariance. The self-energy can be further decomposed as (cf., e.g., Ref. [37])

$$
\gamma_{0} \Sigma(\omega, \mathbf{k})=\Sigma_{+}(\omega, k) \Lambda_{+}(\hat{\mathbf{k}})-\Sigma_{-}(\omega, k) \Lambda_{-}(\hat{\mathbf{k}}),
$$

where

$$
\Lambda_{ \pm}(\hat{\mathbf{k}})=\frac{1 \pm \gamma_{0} \hat{\mathbf{k}} \cdot \gamma}{2}
$$

are projectors on quark states with chirality equal $\left(\Lambda_{+}\right)$or opposite $\left(\Lambda_{-}\right)$to their helicity, and $\Sigma_{ \pm}=b(\omega, k) \pm a(\omega, k)$. The quark propagator then follows as

$$
S(\omega, k) \gamma_{0}=\Delta_{+}(\omega, k) \Lambda_{+}(\hat{\mathbf{k}})+\Delta_{-}(\omega, k) \Lambda_{-}(\hat{\mathbf{k}})
$$

with $\Delta_{ \pm}=-\left[\omega \mp\left(k+\Sigma_{ \pm}\right)\right]$. Since the potential extracted from lQCD is independent of chirality, the self-energy satisfies $\Sigma_{+}=-\Sigma_{-}$; that is $b(\omega, k)=0$. Recalling Eq. (10), we see that this implies that the nonperturbative interactions only contribute to a chirally invariant (thermal) mass term for quarks and antiquarks. For the "gluon-induced" self-energy, $\tilde{\Sigma}$ in Eq. (8), we adopt a form suggested by the high-temperature hard-thermal-loop result, characterized by a mass term, $m$, in the pertinent dispersion relation,

$$
\omega_{k}=\sqrt{k^{2}+m^{2}},
$$

ignoring possible imaginary parts. Our default value for $m$ is $0.1 \mathrm{GeV}$.

Let us now turn to the scattering equation (7). As previously, mentioned, we neglect the (virtual) antiparticle components in the quark propagator and apply a three-dimensional (3-D) reduction scheme to the four-dimensional BS equation, facilitating its numerical evaluation substantially. The resulting Lippmann-Schwinger equation takes the form

$$
\begin{aligned}
T_{a}\left(E ; \mathbf{q}^{\prime}, \mathbf{q}\right)= & V_{a}\left(\mathbf{q}^{\prime}, \mathbf{q}\right)-\int \frac{d^{3} k}{(2 \pi)^{3}} V_{a}\left(\mathbf{q}^{\prime}, \mathbf{k}\right) \\
& \times G_{q \bar{q}}(E ; k) T_{a}(E ; \mathbf{k}, \mathbf{q})\left[1-2 f\left(\omega_{k}\right)\right],
\end{aligned}
$$

where $E$ denotes the center-of-mass (c.m.) energy and $\mathbf{q}$ and $\mathbf{q}^{\prime}$ are the ingoing and outgoing (off-shell) three-momenta in the center of mass (where as usual, the on-shell $T$ matrix is defined by $q=q^{\prime}$ with $E=2 \omega_{q}$, where $\omega_{q}$ is the on-shell single-quark energy); $a=1,8$ labels color-singlet and color-octet channels, and

$$
f(\omega)=\frac{1}{e^{\omega / T}+1}
$$

is the Fermi-Dirac distribution. The explicit form of the twoparticle propagator, $G_{q \bar{q}}(E ; k)$, depends on the 3 -D reduction scheme. Unless otherwise stated, we adopt the BlankenbeclerSugar (BbS) [38], prescription leading to

$$
G_{q \bar{q}}(E ; k)=\frac{\omega_{k}}{\omega_{k}^{2}-E^{2} / 4+2 i \omega_{k} \Sigma_{I}\left(\omega_{k}, k\right)} \quad(\mathrm{BbS}),
$$

but we have checked that our results are very similar when employing the Thompson scheme [39] with

$$
G_{q \bar{q}}(E ; k)=\frac{1}{2} \frac{1}{\omega_{k}-E / 2+i \Sigma_{I}\left(\omega_{k}, k\right)} \quad \text { (Th). }
$$

In both Eqs. (17) and (18) $\omega_{k}$ denotes the on-shell quasiparticle dispersion law, that is, the solution of the equation

$$
\omega_{k}=\sqrt{k^{2}+m^{2}}+\Sigma_{R}\left(\omega_{k}, k\right),
$$

with $\Sigma_{R}$ and $\Sigma_{I}$ the real and imaginary parts of the self-energy. Finally, the potential figuring into Eq. (15) follows from our 1QCD parametrization via Fourier transformation,

$$
V_{a}\left(\mathbf{q}^{\prime}, \mathbf{q}\right)=\int d^{3} r V_{a}(r) e^{i\left(\mathbf{q}-\mathbf{q}^{\prime}\right) \cdot \mathbf{r}} .
$$

To solve Eq. (15) it is convenient to work in a partial-wave basis. Expanding the $T$ matrix and potential,

$$
\begin{gathered}
V_{a}\left(\mathbf{q}^{\prime}, \mathbf{q}\right)=4 \pi \sum_{l}(2 l+1) V_{a, l}\left(q^{\prime}, q\right) P_{l}\left(\mathbf{q}^{\prime} \cdot \mathbf{q}\right), \\
T_{a}\left(E ; \mathbf{q}^{\prime}, \mathbf{q}\right)=4 \pi \sum_{l}(2 l+1) T_{a, l}\left(E ; q^{\prime}, q\right) P_{l}\left(\mathbf{q}^{\prime} \cdot \mathbf{q}\right),
\end{gathered}
$$

allows us to perform the angular integrations to yield

$$
\begin{aligned}
T_{a, l}\left(E ; q^{\prime}, q\right)= & V_{a, l}\left(q^{\prime}, q\right)-\frac{2}{\pi} \int k^{2} d k V_{a, l}\left(q^{\prime}, k\right) \\
& \times G_{q \bar{q}}(E ; k) T_{a, l}(E ; k, q)\left[1-2 f\left(\omega_{k}\right)\right] .
\end{aligned}
$$

In the present study we will constrain ourselves to $S$-wave channels, deferring higher waves to future work. In Ref. [21] it was found that $P$-wave bound-state formation is strongly suppressed (in accordance with our own estimates). Concerning spin-isospin channels, we recall that in the chirally restored phase the spectral functions of chiral partners (e.g., $\left.\pi-\sigma, \rho-a_{1}\right)$ degenerate, which is also reflected in the spectral functions extracted from lQCD [40]. Within the naive constituent quark model, $\pi$ and $\rho$ states are $S$-wave $q-\bar{q}$ bound states, whereas $\sigma$ and $a_{1}$ are in a $P$-wave state. Interestingly, 1QCD spectral functions find an additional (approximate) degeneration of $\pi$ and $\rho$ states above $T_{c}[8,40]$. Insofar as an interpretation of these objects as $q \bar{q}$ states applies, this might be taken as an indication for a spin symmetry much like in heavy-quark effective theories. In view of these considerations, and because our lQCD-extracted potential is flavor blind, we will assume the color-singlet $S$-wave states to appear with a spin-isospin degeneracy corresponding to $\pi+\rho$ states, $d_{\mathrm{SI}}=12$. Since the color-octet potential does not carry any flavor dependence either, the same factor will be applied to the color-octet states.

With the $q-\bar{q} T$ matrix at hand, we can proceed to calculate the explicit expression for the quark self-energy resulting from interactions with anti quarks. Within the imaginary time formalism the latter follows from closing the forward scattering $T$ matrix with a thermal $\bar{q}$ propagator,

$$
\begin{aligned}
\Sigma\left(z_{v} ; p\right)= & \frac{d_{\mathrm{SI}}}{12} d_{a} \int \frac{d^{3} p^{\prime}}{(2 \pi)^{3}}(-T) \\
& \times \sum_{z_{v^{\prime}}} T_{q \bar{q}}^{a}\left(z_{v}+z_{v^{\prime}} ; \mathbf{p}, \mathbf{p}^{\prime}\right) D_{\bar{q}}\left(z_{v^{\prime}}, \mathbf{p}^{\prime}\right),
\end{aligned}
$$


where $z_{v}=\pi i(2 v+1) T$ are fermionic Matsubara frequencies, $d_{1,8}=1,8$ is the color degeneracy factor, and the factor $1 / 12$ represents the average over the $3 \times 2 \times 2$ (color $\times$ flavor $\times$ spin) initial quark states. By using the spectral representations of both the $T$ matrix and $\bar{q}$ propagator to perform the Matsubara sum, and after analytic continuation to the real axis, the self-energy takes the form

$$
\begin{aligned}
\Sigma_{a}(\omega ; p)= & \frac{d_{\mathrm{SI}}}{12} d_{a} \int \frac{d \omega^{\prime}}{2 \pi} \int \frac{d E}{\pi} \int \frac{d^{3} k}{(2 \pi)^{3}} A\left(\omega^{\prime}, k\right) \\
& \times \frac{f\left(\omega^{\prime}\right)+g(E)}{\omega+\omega^{\prime}-E+i \eta} \operatorname{Im} T_{a}(E ; \mathbf{k}+\mathbf{p})
\end{aligned}
$$

with the Bose distribution

$$
g(E)=\frac{1}{e^{E / T}-1}
$$

and the quark spectral function

$$
A(\omega, k)=\frac{-2 \Sigma_{I}(\omega, k)}{\left[\omega-\sqrt{k^{2}+m^{2}}-\Sigma_{R}(\omega, k)\right]^{2}+\Sigma_{I}(\omega, k)^{2}} .
$$

To further simplify our task we assume in the following a quasiparticle approximation for the spectral function,

$$
A(\omega, k)=2 \pi \delta\left(\omega-\omega_{k}\right),
$$

where $\omega_{k}$ is obtained from the self-consistent solution of Eq. (19). (We will check this approximation in the following.) If we furthermore neglect the (weak) energy dependence of $g(E)$ close to the pole of the principal value integral in Eq. (25), we can recover the real part of $T_{q \bar{q}}$ to cast the self-energy in compact form,

$$
\Sigma_{a}(\omega ; p)=\frac{d_{\mathrm{SI}}}{12} d_{a} \int \frac{k^{2} d k d x}{(2 \pi)^{2}}\left[f\left(\omega_{k}\right)+g\left(\omega+\omega_{k}\right)\right] T_{q \bar{q}}^{a}(E),
$$

where $x=\cos \theta$ [with $\theta=\angle(\mathbf{p}, \mathbf{k})]$ and the c.m. energy of the on-shell $T$ matrix is given by

$$
E=\sqrt{\left(\omega_{k}+\omega\right)^{2}-(\mathbf{p}+\mathbf{k})^{2}} .
$$

\section{T MATRIX, SELF-ENERGY, AND SPECTRAL FUNCTION}

In this section we discuss the numerical solutions to the set of equations (19), (23), and (29). Self-consistency is achieved by iteration, starting with the calculation of the $T$ matrix using a constant self-energy in the first step. The self-energy is then calculated from (29) and used to solve the on-shell condition (19). The pertinent quasiparticle dispersion law is then reinserted into the $T$-matrix equation and the procedure is iterated until $T$ matrix and self-energy converge (typically within less than 10 iteration steps). (We have also verified that the final results are insensitive to the initial input value for the selfenergy.)

\section{A. Quark-antiquark $T$ matrix}

The $T$-matrix equation (23) is solved by using the matrix inversion algorithm of Haftel and Tabakin [41] (after discretizing the momentum integration). To assess the possible formation of bound states, the $T$ matrix needs to be calculated below the nominal $q-\bar{q}$ threshold, $E_{\mathrm{thr}}=2[\mathrm{~m}+$ $\left.\Sigma_{R}\left(E_{\mathrm{thr}} / 2,0\right)\right]$. The potential does not depend on the c.m. energy $E$, and, because of its nonrelativistic character, is only defined for real external three-momenta $q$ and $q^{\prime}$. We therefore define the subthreshold on-shell $T$ matrix by setting the external momenta $q=q^{\prime}=0$. In the following we will refer to a peak in the imaginary part of the $T$ matrix as a bound state (resonance) if the energy of the maximum is located below (above) the quasiparticle threshold $E_{\text {thr }}$.

\section{Charmonium systems}

To check the reliability of the parametrization of the potential in the singlet channel, and of the algorithm to compute the $T$-matrix, we first apply our approach to the $c-\bar{c}$ (charmonium) sector by using a (constant) quark mass of $m=1.8 \mathrm{GeV}$, which approximately reproduces the vacuum $J / \psi$ mass at the lowest temperature. (Note that self-consistency does not play a role here since the thermal abundance of $c$ quarks is strongly suppressed; for numerical purposes, we used a fixed imaginary value for the self-energy, $\Sigma_{I}=-10 \mathrm{MeV}$, and $\Sigma_{R}=0$.) The results are displayed in Fig. 4 for three different
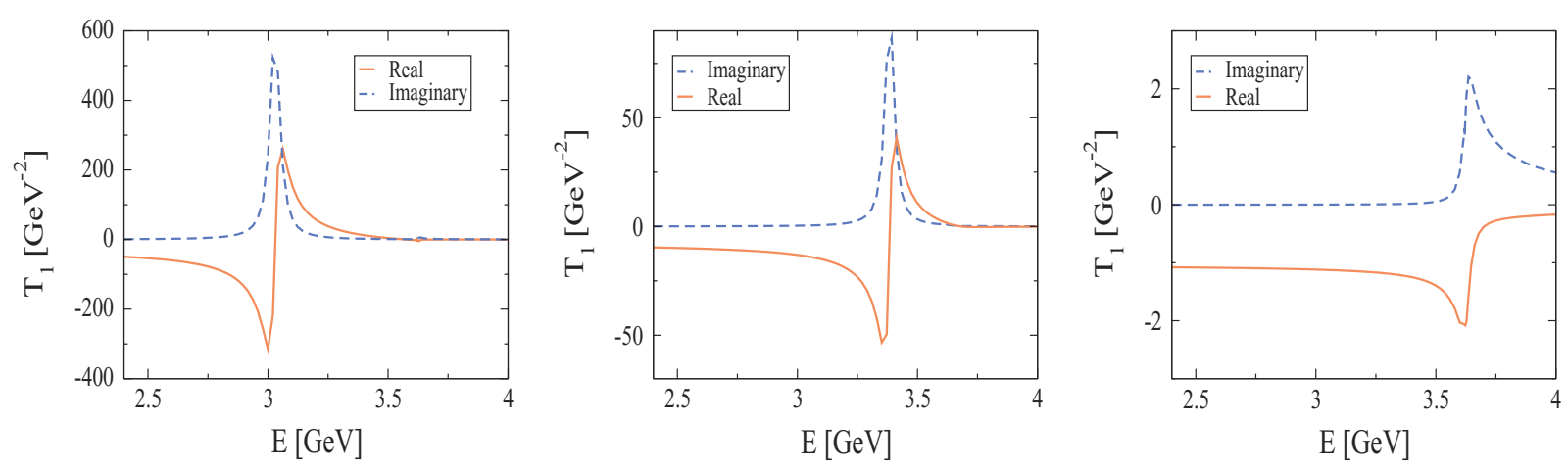

FIG. 4. (Color online) Real part (full red line) and imaginary part (absolute value, dashed blue line) of $T$ matrix in the color-singlet channel for charmonium (with a charm-quark mass of $m=1.8 \mathrm{GeV}$ ) at $T=1.2 T_{c}, T=1.5 T_{c}$, and $T=2 T_{c}$ (left, middle, and right panels, respectively) as a function of c.m. energy $E$, based on our potential parametrization extracted from unquenched lQCD. 

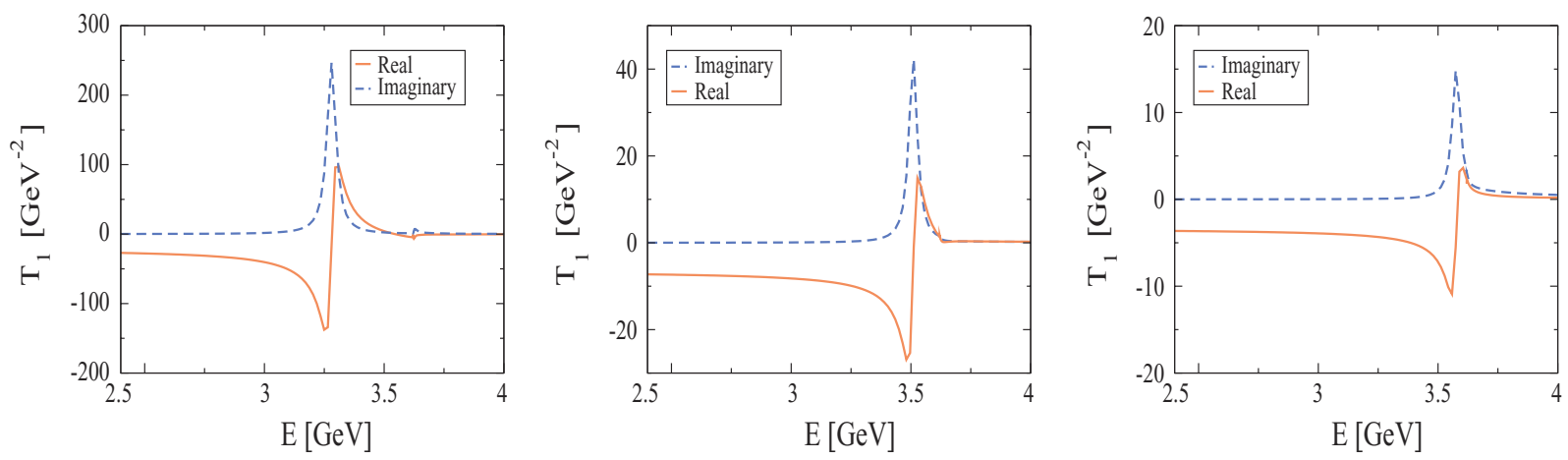

FIG. 5. (Color online) Real part (full red line) and imaginary part (absolute value, dashed blue line) of $T$ matrix in the color-singlet channel for charmonium (with a charm-quark mass of $m=1.8 \mathrm{GeV}$ ) at $T=1.2 T_{c}, T=1.5 T_{c}$, and $T=2 T_{c}$ (left, middle, and right panels, respectively) as a function of c.m. energy $E$, based on a potential [17] extracted from quenched lQCD.

temperatures, $1.2 T_{c}$ (left panel), $1.5 T_{c}$ (middle panel), and $2 T_{c}$ (right panel). As the temperature increases the charmonium state moves up in energy (reflecting a decreasing binding energy), reaching the threshold $\left(E_{\mathrm{thr}}=3.6 \mathrm{GeV}\right)$ at $T \simeq 2 T_{c}$, after which the resonance peak essentially dissolves (Also note that the strength in the $T$ matrix is much reduced at $2 T_{c}$ compared to the lower temperatures.) This behavior is in reasonable (qualitative) agreement with both $1 Q C D$ calculations [6] and effective potential models using a Schrödinger equation $[17,18]$.

To check the sensitivity to the underlying potential (recall the discussion in Sec. II around Fig. 2), we have repeated the calculations for the charmonium $T$ matrix in the singlet channel using the (quenched-based) potential of Ref. [17] (cf. Fig. 5). At the lower temperature of $1.2 T_{c}$ the binding is significantly less pronounced (by about $0.25 \mathrm{GeV}$ ) compared to our parametrization, as to be expected from the less attractive potential. At higher temperatures the agreement improves, and both potentials lead to a very similar temperature where the state crosses the $c-\bar{c}$ threshold (close to $2 T_{c}$ ), with strongly reduced strength. This, in turn, is again in line with the Schrödinger-equation approach, in which the $c-\bar{c}$ system becomes unbound around $\sim 2 T_{c}$ [17]. Although the resonance at $2 T_{c}$ appears to be rather narrow, we recall that we did not include here (temperature-dependent) absorptive parts [10] and reduced masses for the $c$-quarks (nor inelastic charmonium reaction channels [42]), all of which are expected to increase the width of the charmonium states.

\section{Light-quark systems}

For the light-quark sector, the self-consistent results for real and imaginary parts of the on-shell $T$ matrix for quasiparticles with a gluon-induced mass-term of $m=0.1 \mathrm{GeV}$ are summarized in Figs. 6 and 7 for temperatures $T=1.2 T_{c}, T=1.5 T_{c}$, and $T=1.75 T_{c}$.

At $T=1.2 T_{c}$ the color-singlet $T$ matrix exhibits a relatively narrow bound state located significantly below the $q-\bar{q}$ threshold energy of $E_{\mathrm{thr}} \simeq 0.52 \mathrm{GeV}$ (corresponding to twice the real part of the total quark self-energy discussed in the following.) As the temperature increases to $1.5 T_{c}$, the state moves to higher c.m. energy above the threshold ( $E_{\mathrm{thr}} \simeq 0.48 \mathrm{GeV}$ ), which, not surprisingly, is accompanied by a significant broadening. Note also that the peak value is substantially reduced compared to the $1.2 T_{c}$ case, substantially more than would be expected from the broadening alone. We assign this behavior to the decrease in the potential (cf. right panel of Fig. 1), reflecting an overall reduction in interaction strength. The trends in suppression, broadening, and upward energy shift continue at $T=1.75 T_{c}$, where the resonance has now essentially melted,
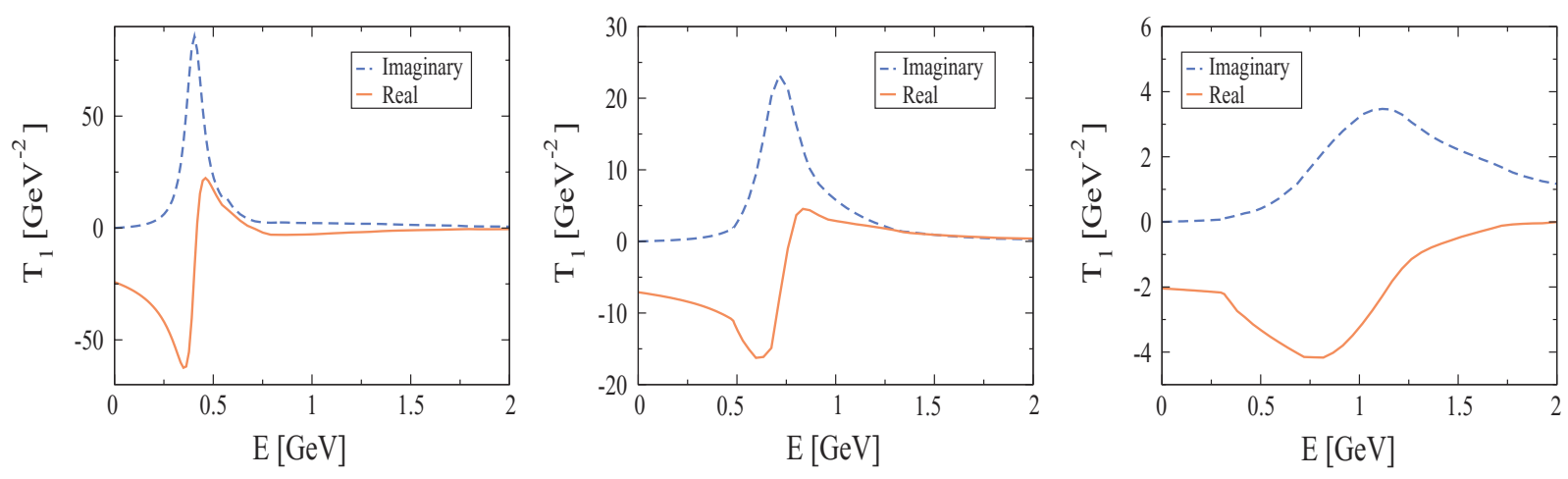

FIG. 6. (Color online) Real part (full red line) and imaginary part (absolute value, dashed blue line) of the light-quark (on-shell) $T$ matrix in the color-singlet channel at temperatures $T=1.2 T_{c}, T=1.5 T_{c}$, and $T=1.75 T_{c}$ (left, middle, and right panels, respectively) as a function of the $q \bar{q}$ c.m. energy $E$, with a "gluon-induced" quark-mass term $m=0.1 \mathrm{GeV}$. 

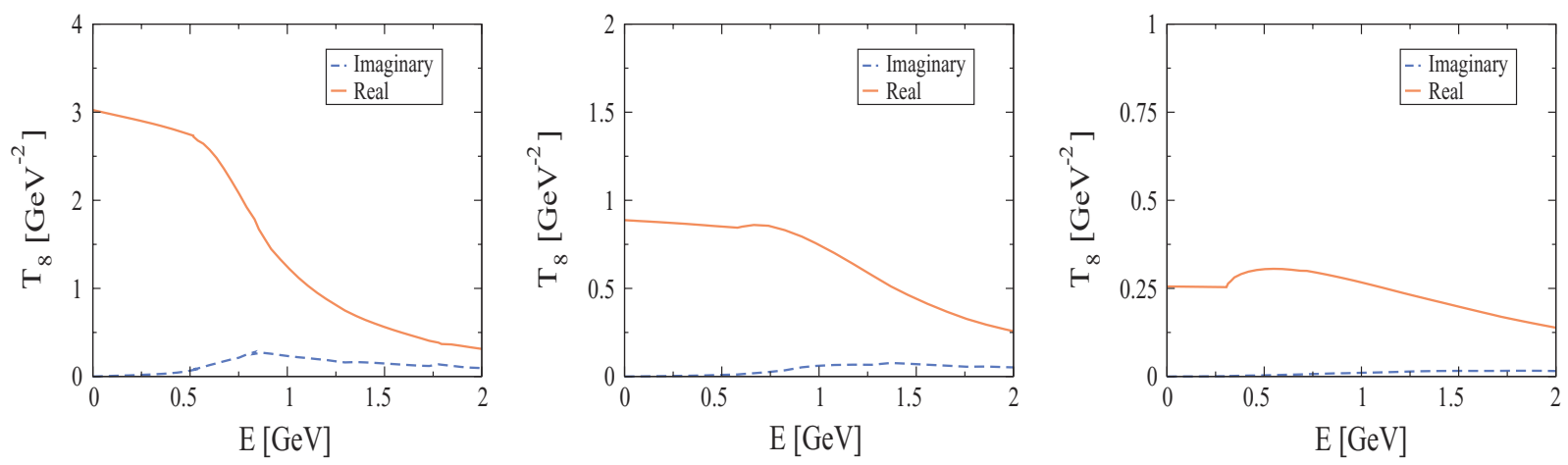

FIG. 7. (Color online) Light-quark $T$ matrix in the color-octet channel vs. $q \bar{q}$ c.m. energy at $T=1.2 T_{c}, T=1.5 T_{c}$, and $T=1.75 T_{c}$ (left, middle, and right panels, respectively) with $m=0.1 \mathrm{GeV}$. Solid (red) line: real part; dashed (blue) line: imaginary part (absolute value).

as indicated by a width of almost $1 \mathrm{GeV}$, comparable to its mass. These results may be put into context with computations of mesonic spectral functions in (quenched) lattice QCD. For (reasonably) light quarks $[8,40]$, their main features above $T_{c}$ are a gradual increase of the peak position (corresponding to the "meson mass") with temperature (roughly proportional to $T$ ), accompanied by a broadening. The bound (resonance) states depicted in Fig. 6 approximately share both of these features.

The $T$ matrix in the color-octet channel is displayed in Fig. 7 for the same set of temperatures. As to be expected for a purely repulsive potential, we find a smooth (nonresonant) dependence of both real and imaginary parts with c.m. energy (with a substantial suppression at higher $T$, as in the singlet case). The imaginary part is very small, and also the real part appears to be small when compared to the singlet channel. We recall, however, that the octet contribution to the self-energy, Eq. (29), enters with a weight that is by a factor of 8 larger than for the singlet one, rendering it an important effect as will be seen below in the following.

\section{B. Self-energy}

We proceed to the single-quark self-energies as calculated from the interactions with antiquarks of the heat bath using the expression, Eq. (29), based on the self-consistent $S$-wave $q-\bar{q} T$ matrices in the " $\pi$ " and " $\rho$ " channels as obtained in the previous section. We recall that the real part of the self-energy corresponds to a chirally invariant mass term, whereas its imaginary part determines the width of a quark (quasiparticle) according to $\Gamma=-2 \operatorname{Im} \Sigma$. Since we work at zero quark-chemical potential, $\mu_{q}=0$, the same results hold for antiquarks. We also recall that our on-shell approximation scheme for the self-energy implies that the effects of bound states are not captured by Eq. (29), since in the integration over the $T$ matrix only energies above the $q-\bar{q}$ threshold, $E_{\mathrm{thr}}$, contribute.

In Fig. 8 the on-shell self-energy is displayed for the same selection of temperatures as in the previous section. Both real and imaginary parts are smooth functions of the quark threemomentum with maximal values at $k=0$. Note that the real part is positive, implying that the repulsive contribution from the octet channels overcomes the attractive singlet channels. The imaginary part (width), in contrast, chiefly arises from resonant scattering in the singlet channel.

More quantitatively, in the temperature regime (1.2-1.5) $T_{c}$, the nonperturbative contribution to the thermal quark mass reaches values of around $150 \mathrm{MeV}$ at small momenta, decreasing to $\sim 50 \mathrm{MeV}$ at $1.75 T_{c}$. With the underlying "gluon-induced" mass term of $m=100 \mathrm{MeV}$, the total thermal mass, $m+\Sigma_{R}$, amounts to $150-250 \mathrm{MeV}$. This is smaller than the effective (perturbative) thermal quark masses required in phenomenological fits to the QGP EoS of 1QCD [33-36]. To improve upon this, we have performed self-consistent
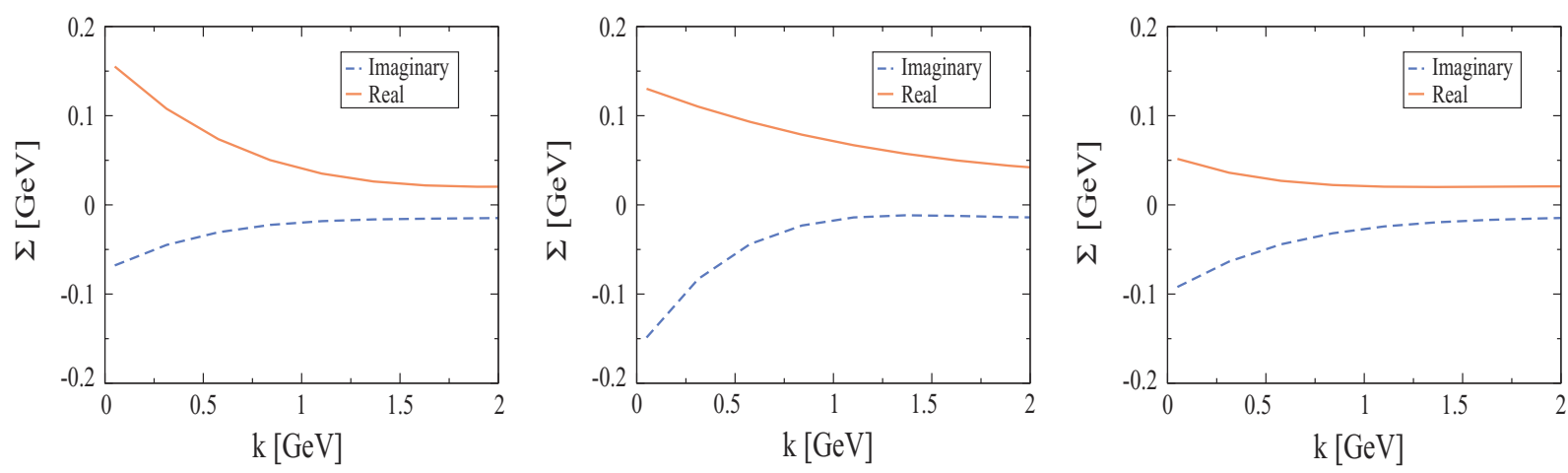

FIG. 8. (Color online) Real part (solid line, red) and imaginary part (dashed line, blue) of the on-shell quark self-energy as a function of three-momentum at temperatures $T=1.2 T_{c}, T=1.5 T_{c}$, and $T=1.75 T_{c}$ (left, middle, and right panels, respectively) with $m=0.1 \mathrm{GeV}$. 

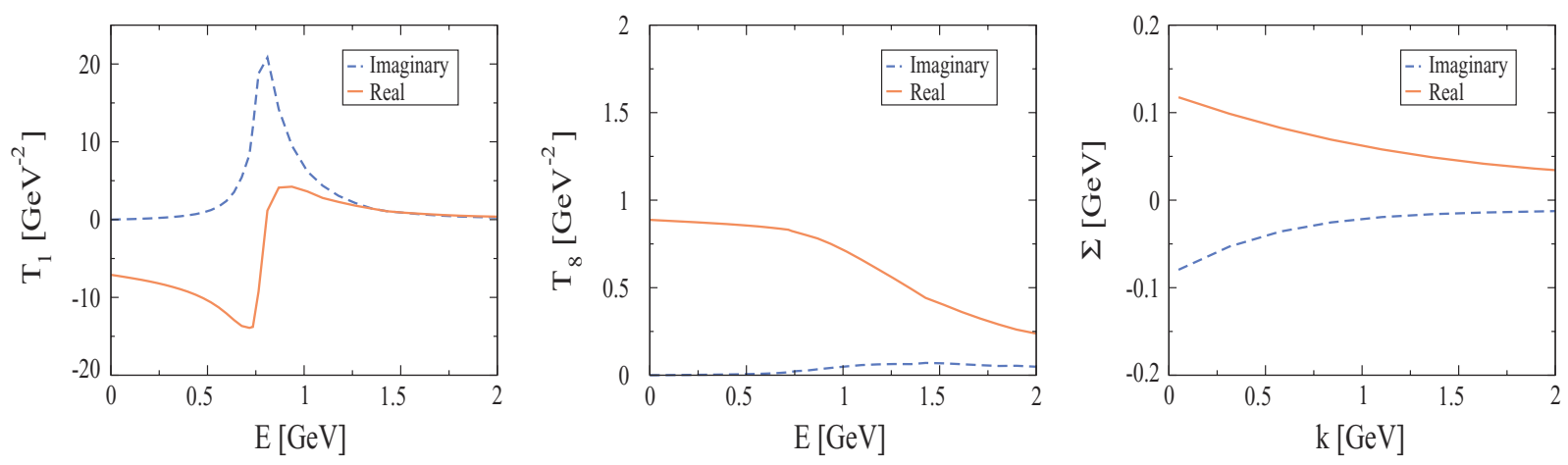

FIG. 9. (Color online) Real part (full line, red) and imaginary part (dashed line, blue) of the $T$ matrix in the color-singlet channel (left panel), color-octet channel (middle panel), and corresponding (singlet + octet) self-energy (right panel) at a temperature $T=1.5 T_{c}$ using a "gluon-induced" mass term of $m=0.25 \mathrm{GeV}$.

calculations with a gluon-induced mass term of $m=250 \mathrm{MeV}$. It turns out that, at given temperature, the "mesonic" states are slightly stronger bound, but in general the behavior of the $T$ matrix and pertinent self-energy are quite similar to those obtained with $m=100 \mathrm{MeV}$. For example, for $T=1.5 T_{c}$ (cf. Fig. 9), the resonance structure is right at threshold, the quark width reaches almost $200 \mathrm{MeV}$, and the combined real part at low momenta amounts to a quark mass of $m+\Sigma_{R} \simeq$ $350 \mathrm{MeV}$.

An important aspect of our results is the rather large imaginary parts of the quark self-energy, translating into widths of about $200 \mathrm{MeV}$ at low momenta for temperatures around $1.5 T_{c}$. As already, mentioned, the width is almost entirely generated by the resonant scattering in the singlet channel; this is nicely illustrated by the significant increase in $\operatorname{Im} \Sigma$ when going from $1.2 T_{c}$ to $1.5 T_{c}$ (cf. left and middle panels in Fig. 8), during which the state in the $T$ matrix moves from below to above threshold (cf. left and middle panels in Fig. 6), that is, converts from bound state to resonance. ${ }^{1}$ The magnitude of the quark widths is quite comparable to the thermal masses, qualitatively supporting the notion that the QGP could be in a liquid-like regime $[43,44]$. Even at the highest considered temperature of $1.75 T_{c}$, and at typical thermal momenta $(k \simeq 3 T \simeq 0.9 \mathrm{GeV})$, the quark width owing to scattering off antiquarks is between 50 and $100 \mathrm{MeV}$. This width does not include $P$-wave interactions, nor strange antiquarks, nor any contributions from scattering off quarks or gluons.

Finally, let us return to the uncertainty associated with the interaction in the octet channel related to the perturbative ansatz, Eq. (6). If the coeffiecient in Eq. (6) is increased (decreased) by a factor 2 (for $T=1.5 T_{c}$ and $m=0.25 \mathrm{GeV}$ ), the imaginary part of the self-energy barely changes, whereas its real part increases (decreases) by about $40 \%$.

\footnotetext{
${ }^{1}$ We recall that bound states are not accessible in on-shell $2 \rightarrow$ 2 scattering; even if a resonance is close to threshold it does not contribute effectively to rescattering processes if the average thermal energy of particles from the heat bath is significant. The contribution of bound states to the self-energy can be included rather by going beyond the quasiparticle approximation, that is, by evaluating Eq. (25) with the off-shell spectral function, Eq. (27).
}

\section{Quark spectral functions and normalization condition}

To better elucidate the validity of the quasiparticle approximation, Eq. (28), we compute the off-shell real and imaginary parts of the self-energy using Eq. (29) and obtain the pertinent quark spectral function $A(\omega, k)$ from Eq. (27). In Fig. 10 we depict $A(\omega, k)$ as a function of quark energy $\omega$ for various fixed momenta at a temperature of $T=1.5 T_{c}$. On the one hand, this reiterates the large effect of the width for low momenta and calls for an off-shell treatment to improve the reliability of our results in the (sub) threshold region of the $T$ matrix. On the other hand, for larger momenta (including typical thermal momenta) the quasiparticle approximation as applied in our calculations appears to be reasonably well justified.

As another check of our approximations we have evaluated the norm of the quark spectral functions defined by

$$
I(k)=\int \frac{d \omega}{2 \pi} A(\omega, k)
$$

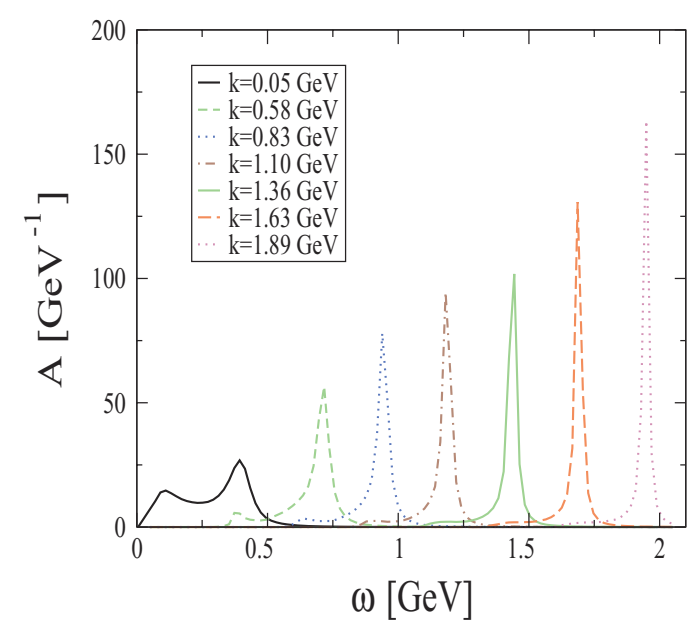

FIG. 10. (Color online) Off-shell spectral function $A(\omega, k)$ as given by Eq. (27) vs. quark energy for different values of the quark momentum at $T=1.5 T_{c}$ and for $m=0.25 \mathrm{GeV}$. 
The unitarity condition for $A(\omega, k)$ requires $I(k)=1$ for each momentum $k$. This relation is rather well satisfied $[I(k) \geqslant 94 \%]$, for all momenta considered in Fig. 10 .

\section{CONCLUSIONS AND OUTLOOK}

In the present article we have set up a self-consistent many-body scheme of Brueckner type to assess nonpertubative properties of (anti-) quarks and mesonic composites in a quark-gluon plasma at temperatures $T \simeq(1.2-2) T_{c}$. Our key ingredient for describing the $q-\bar{q}$ interaction in the QGP was a driving kernel (potential) extracted from unquenched finite- $T$ lattice QCD calculations for the free energy of a heavy-quark pair, supplemented with corrections for relativistic motion. Our main objective was to go beyond earlier applications to bound states by solving the scattering problem thereby accounting for absorptive effects (finite imaginary parts). The self-consistent set of single-quark Dyson and two-body scattering equations has been solved by numerical iteration employing a nonrelativistic reduction of the Bethe-Salpeter equation in connection with a quasiparticle approximation for the quark propagators. One of our main new findings is that the IQCD potentials (dynamically) generate $S$-wave resonance states above the $q-\bar{q}$ threshold up to temperatures of $\sim 2 T_{c}$. These resonances (assumed to occur with a degeneracy corresponding to " $\pi$ " and " $\rho$ " mesons), in turn, play a key role in inducing large quark scattering rates (equal to the imaginary parts of the quark self-energy) as indicated by single-particle widths of $\Gamma \simeq 200 \mathrm{MeV}$ at temperatures around $1.5 T_{c}$. At the same time, significant (positive) real parts arise from repulsive interactions in the color-octet channel entailing thermal masses of up to $\sim 150 \mathrm{MeV}$. We expect that additional contributions to the quark mass of $\sim 250 \mathrm{MeV}$ (induced, e.g., by interactions with thermal gluons as parametrized in quasiparticle models) will be necessary to account for the QGP EoS computed in lattice QCD. Nevertheless, especially at low momenta, the quark widths are comparable to the thermal masses, which could be indicative for liquid-like properties of the QGP at moderate temperatures.

Our analysis suggests several directions for future work. First, the accuracy of our approximations should be scrutinized. This includes improving upon the quasiparticle approximation of the quark spectral function by implementing its off-shell (energy) dependence (as, e.g., carried out in Ref. [45] for a hot pion gas), most notably at low energies to incorporate bound-state contributions to the quark self-energy. The scattering equation ought to be extended to finite total three-momenta of the mesonic composites. Even though we expect the $q-\bar{q}$ channel to constitute a major part of the in-medium interaction, a more complete treatment including $q-q$ and $q-g$ channels is desirable. It is also conceivable that processes of the type $q \bar{q} \rightarrow M g$ (inverse gluon-dissociation, where $M$ is mesonic state) could be significant, as they render bound states accessible in (on-shell) two-body scattering. In a broader context, the underlying EoS of the interacting system needs to be investigated, which is obviously not an easy task. On the phenomenological side, to address the problem of early equilibration at RHIC, it will be of great interest to calculate the thermal equilibration time scales for (anti-) quarks based on the resonant scattering amplitudes found here (e.g., within a Fokker-Planck equation). The elastic scattering rates of around $1 /(\mathrm{fm}) / c$ as found in this work, together with the isotropic angular dependence inherent in $S$-wave rescattering, look promising. For gluons the situation could be more involved since, in addition to bound states as suggested in Ref. [21], other thermalization mechanisms might be operative (e.g., $g g \leftrightarrow g g g$ processes $[46,47])$. In this respect, charm quarks are of particular importance, as their number is presumably frozen after primordial production, and genuine $2 \rightarrow 3$ processes are absent. Indeed, the recent analysis of Ref. [10] has shown that " $D$ "-meson resonances in the QGP can accelerate thermal relaxation times obtained from $\mathrm{pQCD}$ by a factor of $\sim 3$. A rather straightforward extension of our approach to the heavylight sector should therefore be pursued. The formation of mesonic composites in the cooling QGP phase of a heavy-ion collision could furthermore serve as a "pre-hadronization" mechanism and thus improve phenomenologically successful quark-coalescence models at RHIC [48-50] (e.g., with respect to the question of energy conservation). Significant future efforts will be required to possibly develop such a scheme into a quantitative phenomenology. Further progress will also rely on increasing information from finite- $T$ lattice QCD to provide both input and constraints to a many-body approach as presented here. Clearly, a thorough understanding of the intricate properties of the strongly interacting matter above $T_{c}$, and its implications for ultrarelativistic heavy-ion experiments, is an exciting future task.

\section{ACKNOWLEDGMENTS}

We are grateful to P. Petreczky for sending us the data files of the unquenched lattice results for the free energies used in this work. One of us (RR) has been supported in part by a U.S. National Science Foundation CAREER award under Grant No. PHY-0449489. The work of MM has been supported in part by U.S. Department of Energy under Cooperative Research Agreement No. DE-FC02-94ER40818.
[1] R. Baier, A. H. Mueller, D. Schiff, and D. T. Son, Phys. Lett. B502, 51 (2001).

[2] P. F. Kolb and U. Heinz, Quark-Gluon Plasma 3, edited by R. C. Hwa and X. N. Wang (World Scientific, Singapore, 2003).
[3] D. Teaney, J. Lauret, and E. V. Shuryak, Phys. Rev. Lett. 86, 4783 (2001).

[4] T. Hirano, J. Phys. G 30, S845 (2004).

[5] S. Datta, F. Karsch, P. Petreczky, and I. Wetzorke, Nucl. Phys. Proc. Suppl. 119, 487 (2003). 
[6] M. Asakawa and T. Hatsuda, Phys. Rev. Lett. 92, 012001 (2004).

[7] T. Umeda, K. Nomura, and H. Matsufuru, Eur. Phys. J. C 39S1, 9 (2005).

[8] F. Karsch and E. Laermann (2003), hep-lat/0305025.

[9] M. Asakawa and T. Hatsuda, Nucl. Phys. A721, 869 (2003).

[10] H. van Hees and R. Rapp, Phys. Rev. C 71, 034907 (2005).

[11] T. Hatsuda and T. Kunihiro, Phys. Lett. B145, 7 (1984).

[12] T. Hatsuda and T. Kunihiro, Phys. Rev. Lett. 55, 158 (1985).

[13] T. Schäfer and E. V. Shuryak, Phys. Lett. B356, 147 (1995).

[14] X. Li, H. Li, C. M. Shakin, and Q. Sun, Phys. Rev. C 69, 065201 (2004).

[15] W. M. Alberico, A. Beraudo, and A. Molinari, Nucl. Phys. A750, 359 (2005).

[16] S. Digal, P. Petreczky, and H. Satz, Phys. Rev. D 64, 094015 (2001).

[17] C.-Y. Wong, Phys. Rev. C 72, 034906 (2005).

[18] A. Mocsy and P. Petreczky, Eur. Phys. J. C 43, 77 (2005).

[19] E. V. Shuryak and I. Zahed, Phys. Rev. C 70, 021901(R) (2004).

[20] G. E. Brown, C.-H. Lee, M. Rho, and E. Shuryak, Nucl. Phys. A740, 171 (2004).

[21] E. V. Shuryak and I. Zahed, Phys. Rev. D 70, 054507 (2004).

[22] P. Petreczky, F. Karsch, E. Laermann, S. Stickan, and I. Wetzorke, Nucl. Phys. Proc. Suppl. 106, 513 (2002).

[23] O. Philipsen, Phys. Lett. B535, 138 (2002).

[24] O. Kaczmarek, F. Karsch, P. Petreczky, and F. Zantow, Phys. Lett. B543, 41 (2002).

[25] O. Kaczmarek, F. Karsch, F. Zantow, and P. Petreczky, Phys. Rev. D 70, 074505 (2004).

[26] O. Kaczmarek, F. Karsch, P. Petreczky, and F. Zantow, Nucl. Phys. Proc. Suppl. 129, 560 (2004).

[27] P. Petreczky (private communication).

[28] F. Karsch, M. T. Mehr, and H. Satz, Z. Phys. C 37, 617 (1988).
[29] P. Petreczky and K. Petrov, Phys. Rev. D 70, 054503 (2004).

[30] S. Digal, O. Kaczmarek, F. Karsch, and H. Satz, in Proceedings of the International Conference on Hard and Electromagnetic Probes of High-Energy Heavy-Ion Collisions (Ericeira, Portugal, Nov. 4-10, 2004), hep-ph/0505193.

[31] O. Jahn and O. Philipsen, Phys. Rev. D 70, 074504 (2004).

[32] G. E. Brown, Philos. Mag. 43, 467 (1952).

[33] P. Levai and U. Heinz, Phys. Rev. C 57, 1879 (1998).

[34] R. A. Schneider and W. Weise, Phys. Rev. C 64, 055201 (2001).

[35] A. Peshier, B. Kampfer, and G. Soff, Phys. Rev. D 66, 094003 (2002).

[36] J.-P. Blaizot, E. Iancu, and A. Rebhan, Quark Gluon Plasma 3, edited by R. C. Hwa and X.-N. Wang (World Scientific, Singapore, 2003).

[37] U. Kraemmer and A. Rebhan, Rep. Prog. Phys. 67, 351 (2004).

[38] R. Blankenbecler and R. Sugar, Phys. Rev. 142, 1051 (1966).

[39] R. H. Thompson, Phys. Rev. D 1, 110 (1970).

[40] M. Asakawa, T. Hatsuda, and Y. Nakahara, Nucl. Phys. A715, 863 (2003).

[41] M. I. Haftel and F. Tabakin, Nucl. Phys. A158, 1 (1970).

[42] L. Grandchamp, R. Rapp, and G. E. Brown, Phys. Rev. Lett. 92, 212301 (2004).

[43] A. Peshier and W. Cassing, Phys. Rev. Lett. 94, 172301 (2005).

[44] M. H. Thoma, J. Phys. G 31, L7 (2005).

[45] R. Rapp and J. Wambach, Phys. Lett. B351, 50 (1995).

[46] S. M. H. Wong, Nucl. Phys. A607, 442 (1996); Phys. Rev. C 54, 2588 (1996).

[47] Z. Xu and C. Greiner, Phys. Rev. C 71, 064901 (2005).

[48] R. J. Fries, S. A. Bass, and B. Muller, Phys. Rev. Lett. 94, 122301 (2005).

[49] V. Greco, C. M. Ko, and P. Levai, Phys. Rev. Lett. 90, 202302 (2003).

[50] R. C. Hwa and C. B. Yang, Phys. Rev. C 67, 034902 (2003). 